\title{
A profile of patients with substance use disorders and treatment outcomes: A 10-year retrospective study from the National Rehabilitation Center
}

Ahmed Elkashef ${ }^{\text {, }}$ Taoufik Zoubeidi², Rekha Ann Thomas ${ }^{3}$, Hanan Al Hashmi, Amanda J Lee ${ }^{4}$,Tar-Ching Aw ${ }^{3}$, Ian Blair ${ }^{3}$, Hisham F Al Arabi ${ }^{1}$, Hamad Alghafri ${ }^{1}$

${ }^{1}$ National Rehabilitation Center, Abu Dhabi, United Arab Emirates; ${ }^{2}$ Department of Statistics, UAE University, Al Ain, United Arab Emirates; ${ }^{3}$ Institute of Public Health, College of Medicine \& Health Sciences, UAE University, Al Ain, United Arab Emirates; ${ }^{4}$ Division of Applied Health Sciences, University of Aberdeen.

\section{Abstract}

\section{Objective}

The National Rehabilitation Center (NRC) is the major center in the United Arab Emirates (UAE) treating patients with substance use disorders (SUD). The objective of our study is to analyze data from the NRC to examine the trends of SUD in the UAE nationals over the last 10 years and to assess the status of the treatment programme at the NRC.

\section{Methods}

Data was collected during a 10 year period from patients' records admitted to the NRC (from its inception in 2002 to 2011) to study socio-demographic characteristics, patterns of SUD as well as co-morbid conditions.

\section{Results}

A total of 591 patients were assessed and/or admitted to the NRC during this period. All patients were males between the age group $16-66$ years, forty two percent were married, $60 \%$ were unemployed and $51 \%$ were educated below secondary school level. Seventy seven percent of the patients were admitted voluntarily and $23 \%$ were involuntarily admitted. Forty one percent was admitted due to alcohol consumption, $20 \%$ for prescription drugs and $16 \%$ for heroin.

\section{Discussion}

Relapse rate and retention in treatment were used as indicators of the effectiveness of the treatment programmes at the NRC. The number of admissions per patient each year was used as an estimate of relapse which showed a drop from about $60 \%$ in the first two years when the NRC opened to about $20 \%$ recently. Rates of retention/completion of inpatient treatment and discharge to outpatient care, half-way house or home were about $73 \%$ 
with $27 \%$ of patients terminating their treatment earlier. Overall, there has been a steady increase in SUD over the last 10 years with increasing trends of prescription drugs and polysubstance addiction among youth. This necessitates expansion of services to meet the increasing demands as well as increasing efforts towards nationwide effective prevention and public awareness approaches.

Keywords: Voluntary; Involuntary: Addiction; Polysubstance; abuse; Relapse

\section{Introduction}

The prevalence of substance use disorders (SUD) assessed by the United Nations Office on Drugs and Crime (UNODC) Drug Report ${ }^{1}$ claims that it is steadily increasing globally and it continues to be a problem impacting public health. Degenhardt ${ }^{2}$ states that compared to the global data, the Middle Eastern region has a considerably smaller proportion of its population abusing alcohol or drugs. However, this can be partially attributed to the lack of statistics collected or limited studies conducted in these areas. It can also be partly due to religious influences and strict laws prohibiting the use and supply of alcohol and illicit substances in the Middle East.

Due to these restrictions, SUD are usually confidentially dealt within concealed environments that may not be conducive for in-depth research to study the extent and contributory factors for alcohol and drug misuse, and studies on effective interventions and prevention.

AbuMadini compared data of the drug treatment services in Saudi Arabia, from 1986-1996, which showed a 25\% increase in the mean number of drugs abused per individual (from 1.3 to 1.6 ) in a decade. ${ }^{3}$ There was also a shift from alcohol as the sole substance of abuse to amphetamine-type stimulants in the form of fenethylline (captagon), with increasing numbers of instances of confiscations by enforcement authorities being reported by UNODC. ${ }^{1} \mathrm{Al}$ Kandari in Kuwait showed that the number of patients admitted for addiction treatment rose from 386 in 1997 to 779 in $1999 .{ }^{4}$

The United Arab Emirates (UAE) comprises seven Emirates with a total population estimated at 8,264,070, as recorded by Population Statistics of which only $11.4 \%$ are Emirati citizens and $88.6 \%$ are expatriates. ${ }^{5}$ The Emirate of Abu Dhabi is the largest with a total population of 2,422,400 as listed in Health Authority Abu Dhabi. ${ }^{6}$

Reports on drug abuse in the UAE are limited. Multiple studies (7-8) from the UAE speculate that dramatic social changes as well as a breakdown in religiosity could be contributing factors for the increase in drug abuse amongst the Emirati population. SUD are known to cause significant mortality and morbidity. Yet, proper data about drug abuse in the UAE, the extent and magnitude of health effects are scarce. 
Amin's study using data derived from a psychiatric practice in the UAE showed that heroin abuse was more than alcohol abuse. ${ }^{7}$ According to recent data, Amir indicates that about $42.3 \%$ of users are addicted to more than one substance. ${ }^{9}$

A recent report by Al Marri from another UAE psychiatric hospital stated that within a two year period (1990-1991), 9.5\% of its patients were admitted for SUD. ${ }^{10}$

Patients are seen at the NRC for treatment either voluntarily (self-referrals) or involuntarily following a requirement by the court system, police, families or an employer. Patients undergo an initial assessment by a team of professionals comprising of social workers, psychologists and psychiatrists for admission. A brief admission of up to two weeks could be the part of the initial assessment. Based on the initial assessment patients are either admitted to the NRC as in-patients or referred to other centers if they have an exclusionary medical or psychiatric condition, or given advice and counselling but not admitted for inpatientservice. Since the opening of the outpatientservices attheNRC, patients who do not need inpatient treatment are admitted to the outpatient services.

Inpatient treatment was the only treatment setting available at the NRC till 2009 till the outpatient clinic opened. Admitted patients have an in-depth psychiatric assessment, psychological tests, medical examination and social assessments and a comprehensive diagnosis which helps medical personnel to prepare a treatment plan specific for each patient. During the inpatient stay, the treatment plan could include detoxification, medications and behavioural therapy in the form of individual or group therapy. Patients also undergo urine drug screening on a regular basis to ensure compliance. Upon completion of in-patient treatment patients are discharged to outpatient treatment, a half-way house to continue their treatment. Others are discharged home or returned to jail.

\section{Objective}

The objective of our study is to analyse data from the NRC to examine the trends of SUD in the UAE nationals over the last 10 years and to assess the status of the treatment programme at the NRC. Located in Abu Dhabi, the $\mathrm{NRC}$ is an independent comprehensive center for substance abuse providing inpatient and outpatient treatment, rehabilitation services and educational awareness serving the UAE. This establishment is the only center providing such services in the nation and caters for Emirati nationals. 


\section{Methods}

The study included data on all patients assessed and admitted to the NRC from the opening of the center in 2002 till 2011. The study was reviewed and approved by the human ethics committee at the United Arab Emirates University. All data were collected confidentially using file numbers with no personal identifiers (e.g. name or address).

Case notes, monthly summary sheets and paper records of all patients assessed were trawled to extract information for several parameters, e.g. demography, nature and pattern of drug use, diagnosis, educational level, referral source and discharge plans, family history of alcohol or drug misuse as well as other mental or medical co-morbidities. Clinical staffs of the NRC were consulted to resolve missing data, unclear information, or conflicting statements in the records.

A $50 \%$ random sample of cases was checked by a different independent researcher with regard to accuracy and consistency of information. If a discrepancy was detected, it was resolved through discussion between two researchers, or else, a third independent person was consulted.

Information collected was entered into a Microsoft Access computer database and then exported to the SPSS statistical software package for analysis.

Appropriate descriptive statistics were used to summarize the data such as medians and Inter-Quartile Range statistics (IQR) to summarize skewed data. The independent samples t-test was used to compare groups of patients on their first admission or assessment with respect to quantitative variables and Pearson's chi-square test was used to examine associations among categorical variables at the time of the first admission or assessment episode.

\section{Results}

\section{Patient demographics}

Data were available for 834 patient admission records representing a total of 591 patients (some patients were admitted at the NRC more than once).

Of the 591 patients, only 221 were assessed or admitted briefly up to two weeks for the purpose of further evaluation to determine the suitability for long term admission. A total of 369 were admitted for treatment. There was insufficient information for two patients and they have been excluded from further analysis.

All patients were Emirati males with, at first admission, an average age of 32.4 years $(\mathrm{SD}=9.6)$. Forty four percent were single, $42 \%$ married and $13 \%$ were divorced (Table 1). Sixty percent of patients were unemployed and 33\% were either employed or students. Fifty one percent did not have a secondary 
education, $33 \%$ had secondary education and $16 \%$ had a post-secondary education.

Seventy seven percent $(n=607)$ of the patients were admitted voluntarily and $23 \%(n=182)$ were involuntary patients.

The family history report indicated that at first admission $155(30 \%)$ of patients were from families with a history of alcohol abuse and $84(16 \%)$ were from a family with a history of drug abuse.

Depending on the patient's assessment and the treatment plan, the minimum recommended inpatient stay at the NRC ranged from 6-12 weeks. The median (IQR) admission duration was 64 (IQR 153-21) days. However, the median inpatient duration of the voluntary group was 34 (IQR 91-11) days and were substantially lower than that of the involuntary admissions of 107 (IQR 185523) days.

Total number of admissions to the NRC increased from the first quarter of 2002 to third quarter of 2011, with a steeper increase since 2009. The observed increase is due to the voluntary admissions, while the involuntary referrals have remained stationary from 2002 to 2011 (Figure 1).

Figure 1. Numbers of admission or assessment episodes by type of referral

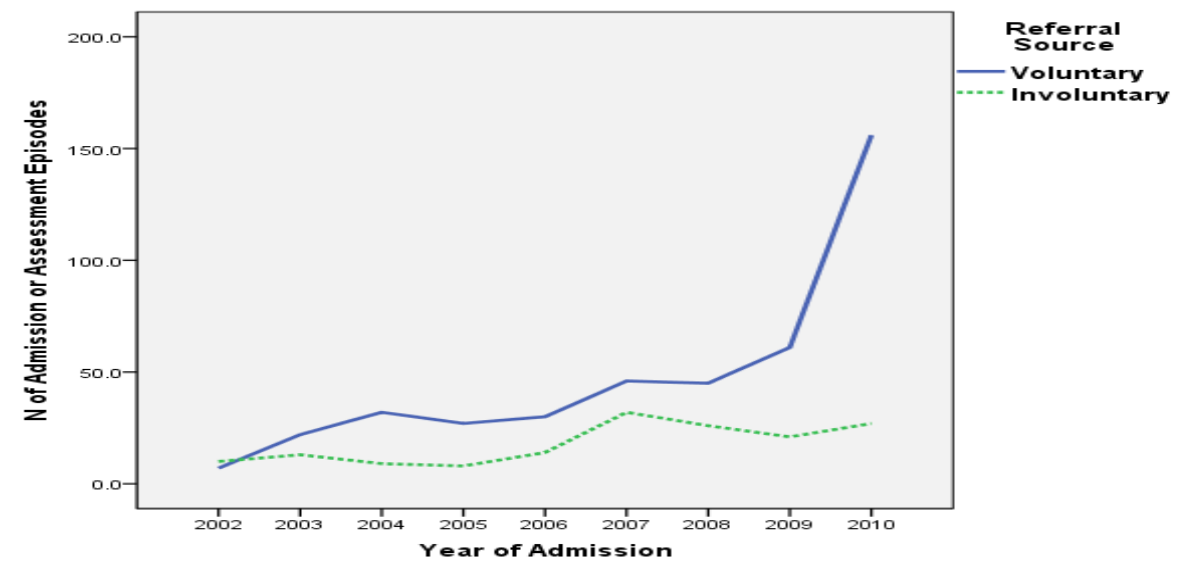


Table 1. Patient demographics at the time of the first referral to NRC

\begin{tabular}{|c|c|c|}
\hline & Count & Percent \\
\hline \multicolumn{3}{|l|}{ Marital Status } \\
\hline Married & 243 & 42.0 \\
\hline Single & 253 & 43.7 \\
\hline Divorced & 76 & 13.1 \\
\hline Widowed & 2 & 0.3 \\
\hline Separated & 5 & 0.9 \\
\hline Total & 579 & \\
\hline \multicolumn{3}{|l|}{ Residence } \\
\hline Abu Dhabi City & 262 & 45.8 \\
\hline Al Ain & 133 & 23.3 \\
\hline Dubai & 56 & 9.8 \\
\hline Ajman & 27 & 4.7 \\
\hline Sharjah & 66 & 11.5 \\
\hline RAK & 12 & 2.1 \\
\hline Fujairah & 8 & 1.4 \\
\hline Umm-Al-Qawain & 5 & 0.9 \\
\hline Other & 3 & 0.5 \\
\hline Total & 572 & \\
\hline \multicolumn{3}{|l|}{ Employment Status } \\
\hline Employed/ Student* & 191 & 33.3 \\
\hline Unemployed & 346 & 60.3 \\
\hline Retired & 37 & 6.4 \\
\hline Total & 574 & \\
\hline \multicolumn{3}{|l|}{ Education Status } \\
\hline Illiterate & 9 & 1.6 \\
\hline Primary School & 116 & 20.9 \\
\hline Middle School & 157 & 28.3 \\
\hline Secondary School & 183 & 33.0 \\
\hline $\begin{array}{l}\text { Post-secondary } \\
\text { education }\end{array}$ & 90 & 16.2 \\
\hline Total & 555 & \\
\hline
\end{tabular}


* The figure does not total to 591 subjects due to missing data

* This category cannot be separated because they were entered as one category in the data.

The age distribution, marital and employment status were similar for both voluntary and involuntary patients. The mean (standard deviation) age of voluntary and involuntary referrals were, 32.0 (9.5) years and 33.6 (10.3) years respectively ( $p$-value $=0.085$ ). Similar profiles with respect to marital and employment status in the first episode were also noted ( $p$-values of Pearson's chi-square test $=0.902$ and 0.726 , respectively).

There were differences between these groups with respect to educational level with $67 \%$ of voluntary and $47 \%$ of involuntary admissions having intermediate or secondary education. However, only $14 \%$ of voluntary referrals had postsecondary education compared to $22 \%$ of involuntary patients $(\mathrm{p}=0.019)$.

\section{Drug Use Pattern}

The main reported substance of abuse was alcohol $41.3 \%$, whereas, other drugs such as tetrahydrocannabinol (THC), benzodiazepines, amphetamines and inhalants were $22.5 \%$ and heroin $16.3 \%$. The remaining drugs included prescription drugs, e.g. pain killers such as tramadol, methadone, codeine, sedatives such as xanax and valium, and other psychoactive substances such as kemadrine, artane and khat. Since 2009, there has been a drastic increase in the use of prescription drugs and other psychoactive polysubstance abuse than alcohol, heroin and THC abuse (Figure 2).

Figure 2. Distribution of the main drug of abuse over time

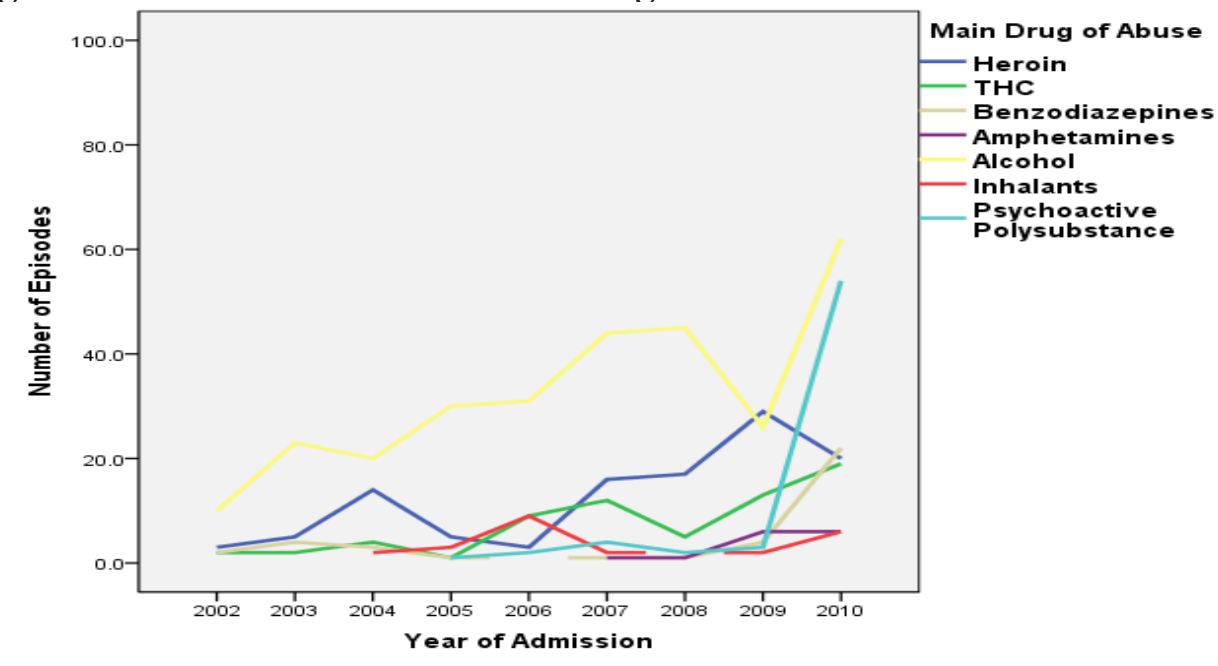


Heroin and other drugs were the preferred choice for younger patients aged 1626 years, while alcohol was favoured among those aged 37-66 years (Figure 3). Poly-substance use (abuse of three or more substances simultaneously) steadily increased over time with a drastic increase since 2009.

Drug use pattern for voluntary and involuntary admissions were $47 \%$. Of this, $39 \%$ was for alcohol abuse for both voluntary and involuntary admissions. However, abuse of other psychoactive substances was three times more for voluntary patients $(24 \%$ and $7 \%$ respectively). On the other hand, the percentage of abusers of THC among involuntary patients was approximately double that for voluntary patients ( $19 \%$ and $8 \%$, respectively).

\section{Figure 3. Distribution of main drugs of abuse by age group}

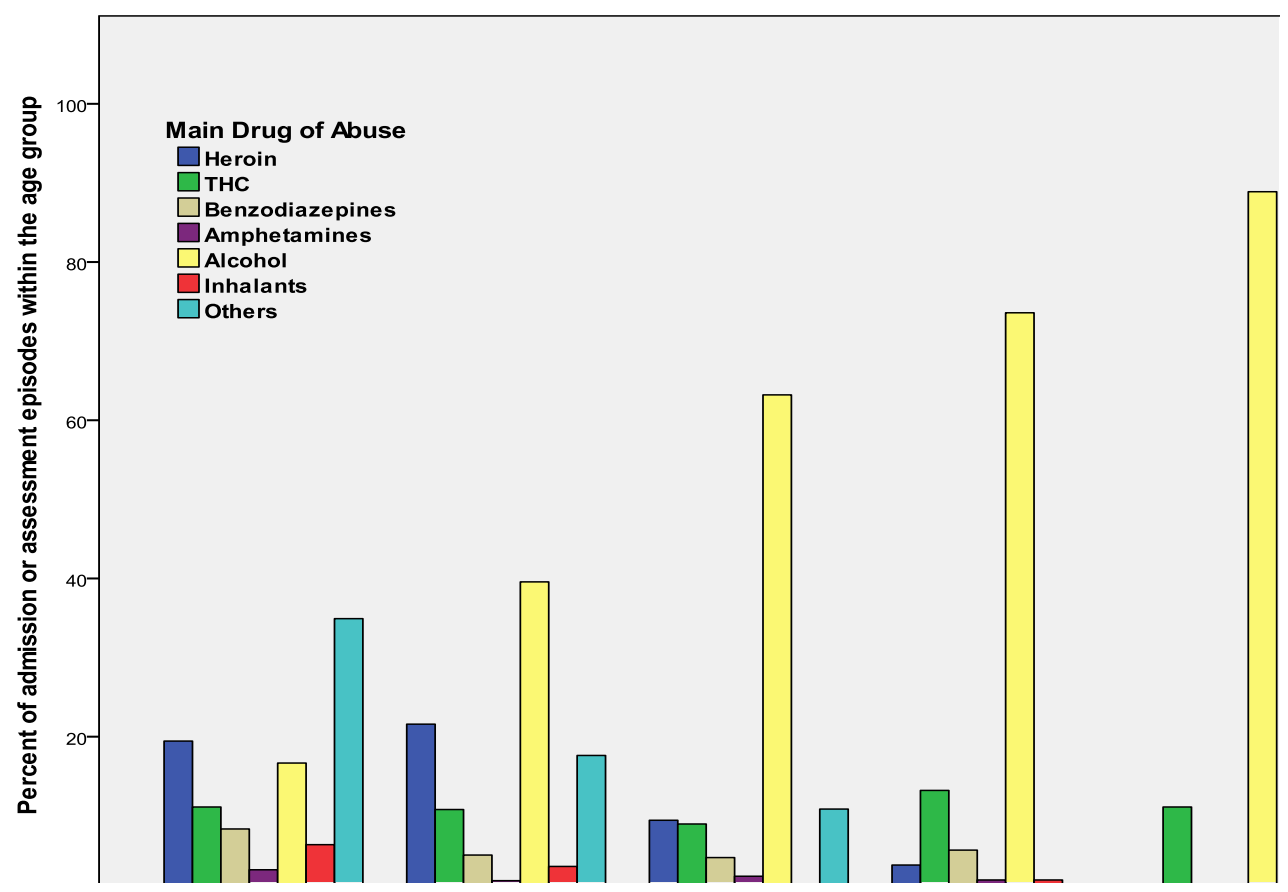

\section{Co-morbid conditions}

The initial medical screening for all referred patients included determination of Hepatitis B and C carrier status. Among those screened, 3\% $(n=14)$ and $15 \%(\mathrm{n}=80)$ patients were positive for Hepatitis B and Hepatitis C respectively. Amongst intravenous drug users, the prevalence of Hepatitis C was $44 \%$ compared to $11 \%$ amongst those who mainly inhaled drugs.

A wide range of psychiatric disorders were grouped under three prevalent categories such as anxiety disorders, mood disorders and other psychotic disorders (Table 2). The highest prevalence of these psychiatric conditions was observed in $25 \%$ of patients with alcohol abuse, $16 \%$ in those with polysubstance abuse and $13 \%$ in THC abusers. 
Table 2: Distribution of psychiatric disorders among substance disorders

\begin{tabular}{|c|c|c|c|c|c|}
\hline \multirow{2}{*}{\multicolumn{2}{|c|}{ Substance Related Disorders }} & & \multicolumn{2}{|c|}{$\begin{array}{c}\text { Psychiatric } \\
\text { Disorder }\end{array}$} & \multirow[b]{2}{*}{ Total } \\
\hline & & & No & Yes & \\
\hline \multirow{4}{*}{$\begin{array}{l}\text { Polysubstance related } \\
\text { disorders }\end{array}$} & No & & & & \\
\hline & & Percent & 75.6 & 24.4 & 100 \\
\hline & Yes & & & & \\
\hline & & Percent & 84.2 & 15.8 & 100 \\
\hline \multirow{4}{*}{$\begin{array}{l}\text { Alcohol only related } \\
\text { disorders }\end{array}$} & No & & & & \\
\hline & & Percent & 83.3 & 16.7 & 100 \\
\hline & Yes & & & & \\
\hline & & Percent & 75.4 & 24.6 & 100 \\
\hline \multirow{4}{*}{$\begin{array}{l}\text { Opioid only related } \\
\text { disorders }\end{array}$} & No & & & & \\
\hline & & Percent & 81.1 & 18.9 & 100 \\
\hline & Yes & & & & \\
\hline & & Percent & 100 & 0.0 & 100 \\
\hline \multirow[t]{3}{*}{$\begin{array}{l}\text { Cannabis only related } \\
\text { disorders }\end{array}$} & No & Percent & 81.4 & 18.6 & 100 \\
\hline & Yes & & & & \\
\hline & & Percent & 86.7 & 13.3 & 100 \\
\hline \multirow{4}{*}{$\begin{array}{l}\text { Inhalant only related } \\
\text { disorders }\end{array}$} & No & & & & \\
\hline & & Percent & 81.4 & 18.6 & 100 \\
\hline & Yes & & & & \\
\hline & & Percent & 90.9 & 9.1 & 100 \\
\hline \multirow{4}{*}{$\begin{array}{l}\text { Sedative hypnotic or } \\
\text { anxiolytic only related } \\
\text { disorders }\end{array}$} & No & & & & \\
\hline & & Percent & 81.8 & 18.2 & 100 \\
\hline & Yes & & & & \\
\hline & & Percent & 0.0 & 100 & 100 \\
\hline \multirow{4}{*}{$\begin{array}{l}\text { Stimulant only related } \\
\text { disorders }\end{array}$} & No & & & & \\
\hline & & Percent & 81.5 & 18.5 & 100 \\
\hline & Yes & & & & \\
\hline & & Percent & 88.9 & 11.1 & 100 \\
\hline \multirow{4}{*}{$\begin{array}{l}\text { Other substance only } \\
\text { related disorders }\end{array}$} & No & & & & \\
\hline & & Percent & 81.5 & 18.5 & 100 \\
\hline & Yes & & & & \\
\hline & & Percent & 100 & 0.0 & 100 \\
\hline
\end{tabular}




\section{Programme outcomes}

In order to assess the effectiveness of the administered treatments at the NRC we examined four outcomes. The first was treatment retention as evidenced by completion of the proposed treatment and transfer of patients from one programme to another, e.g. from inpatient to outpatient. We also examined the relapse rate among patients discharged from treatment and the availability of medications to treat SUD.

\section{a) Treatment retention}

The overall treatment retention rate was estimated to be about $71 \%$, where $66.3 \%$ of patients were transferred to the outpatient clinic or to the residential program $(0.6 \%)$ and $4.7 \%$ of such patients returned home after successful treatment. About $20 \%$ of patients left against medical advice, $4.1 \%$ had a disciplinary discharge, and $2.5 \%$ returned to jail.

\section{b) Relapse data}

The only available recorded data as a possible indication of a patient's relapse was the number of readmissions per patient. Prior to the establishment of the outpatient services in 2009, there were no long term follow up plans for patients who completed their inpatient treatment and were discharged later. This is not the most accurate way of calculating relapse or ascertaining lapses, as the calculation was based on the number of admissions per patient from the end of a treatment session to a subsequent admission with documented drug use. Of 589 patients, 221 $(38 \%)$ were not actually admitted. A total of $268(46 \%)$ had only one admission, $10 \%$ were admitted twice and $7 \%$ were admitted three times or more. Among the 368 admitted patients, 100 were re-admitted at least once which represents a crude relapse rate of $27.2 \%$.

The NRC reports a reduction from a 60\% relapse rate in 2003 (one year after the center opened) to a $20 \%$ relapse rate in 2010 .

Half the patients who relapsed had an average duration to relapse (duration between successive admissions) of 159 days or more, and one quarter of the relapsed patients had an average duration to a relapse of 25 days. The median (IQR) duration stay of patients at the NRC was 64 days (153-21) in the first admission, 26 days (85-9) in the second admission and 37 days (92-7) in the third admission.

The median (IQR) duration to relapse for the voluntary and involuntary patients was 106 (295-22) days and 149 (725-27) days respectively. 
The type of admission was compared between voluntary and involuntary admissions upon readmission to the NRC. Ninety one percent of those who were admitted on voluntary basis at first episode were re-admitted voluntarily in the second admission episode whereas $71 \%$ of those who were admitted on an involuntary basis in the first episode were readmitted on a voluntary basis in the second admission.

\section{c) Use of medication for SUD}

Data was collected on the rate of prescribing medications for SUD, mainly suboxone, subutex, acamprosate, disulfiram and naltrexone. Many patients received other medications for co-morbid psychiatric conditions or for symptomatic relief. Yet, this data was not extracted.

In $70 \%(\mathrm{n}=559)$ of the total 834 admission or assessment episodes, no medications were prescribed. This percentage dropped to $58 \%$ when considering the 539 admission episodes only. A single medication was prescribed in $31 \%(\mathrm{n}=166)$ of the admission episodes, two medications in $10 \%(n=54)$ of the admissions, and three medications in $1 \%(n=5)$ of admissions.

Acamprosate was prescribed for $19 \%(\mathrm{n}=100)$ of the 537 admission episodes. Suboxone was prescribed in $15 \%(\mathrm{n}=80)$ of admissions, and naltrexone in $6 \%$. When medication was prescribed after diagnosis, suboxone was prescribed for $31 \%(n=31)$ of the 101 admissions where heroin was the main drug abused and for $42 \%(n=26)$ of the 62 admissions for other types of opioid prescription drugs. Acamprosate was prescribed for $36 \%(n=89)$ of the 246 admissions where alcohol was the main drug abused.

\section{Discussion}

This was an exploratory exercise with the main goal of getting some idea of drug use trends during the last 10 years in the UAE and is a collection of patients' profiles and some outcome measures to gauge the effectiveness of the treatment programs at the NRC over time. There was no a priori hypothesis or expectations that were tested. Despite the cultural, social and religious norms of the Arab society, a number of studies conducted in the Middle Eastern Region, mainly in Saudi Arabia, Kuwait, Oman and the UAE, reports of an increase in alcohol and drug abuse. Reasons for such increase are peer group influences, family history, overseas travel and curiosity for experimentation with drugs. ${ }^{10,11}$ 
The results of this study are consistent with these reports and also show a steady increase in the number of admissions with a sharp increase in admissions since 2009. This rise is attributed to an increase in the number of treatment seekers, expansion of services around that time to include an outpatient clinic and a half way house and increased awareness through media campaigns.

Studies of risk factors for drug use, especially among the younger age groups are currently underway, to better explain the drastic increase in drug abuse and to design appropriate preventive measures to curb this menace.

The patterns of drug use have changed from mostly single substance abuse with alcohol, heroin and marijuana to prescription drug use and polysubstance abuse especially among those aged 16-26 years. Hafeiz observes a similar trend in two studies conducted in Saudi Arabia where 27\% reported abusing more than one drug in 1992 and 37\% patients using a combination of drugs in a later study in $1995 .{ }^{12}$

Inpatient treatment was the main and only treatment available at the NRC between 2002 and 2009 when the outpatient services were added. A total of $66 \%$ of the inpatients continued to receive treatment after being discharged as outpatients. This has probably contributed to the decrease in relapses noted during recent times. The overall retention rate would suggest a good compliance with the short term and long term treatments recommended. Measures of motivation, impulsivity and satisfaction with treatment will help elucidate factors contributing to this compliance and retention in treatment. Involuntary admissions have remained steady for the last 10 years around one quarter of the total admissions. However, involuntary patients have longer average stays than voluntary patients. The reason for the longer stay is that court ordered patients are usually sent to the NRC for inpatient treatment usually for six months. These court orders are non-negotiable, despite the fact that patients were considered as suitable candidates for a shorter duration of treatment or for outpatient treatment. These issues with the legal system in the UAE and courts handling drug abuse cases are being discussed to avoid such shortcomings.

The 10 year relapse trends at the NRC have shown a marked drop from an initial $60 \%$ when it was first opened to about $20 \%$ in $2010 / 2011$. This decrease can possibly be due to the patient specific treatment effect, better treatment focusing on cognitive behavioural therapy and relapse prevention. Group therapy and support groups like Alcoholics Anonymous (AA) were also recently introduced in the UAE and many patients were encouraged to join these groups after being discharged or while enrolled for outpatient treatment. 
In a study exploring relapse rates in Saudi Arabia, Al-Nahedh reported that $60 \%$ of its patients were admitted more than once mainly attributing it to social stress and unemployment. ${ }^{13}$ Similarly, Rahim reports a hospital record of relapse rate of $59.7 \%$ with $90 \%$ occurring within the first 42 months of discharge. ${ }^{14}$

Data on re-admissions showed that $71 \%$ of the involuntary patients when admitted for relapse, seek treatment voluntarily. This could be a treatment effect from previous admissions which has resulted in reduced criminal or anti-social activity or possibly because patients learned better ways to avoid the problem avoid being caught by authorities for drug abuse.

Infectious disease rates of hepatitis $\mathrm{C}$ are somewhat on the low side due to lower rates of intravenous drug use. HIV data are not available since HIV positivity is an exclusion criteria for NRC admittance. Co-morbid conditions were also lower than reported internationally. This could be due to under diagnosis or incorrect reporting of facts. Measures to standardise and improve diagnosis of mental disorders like the use of the Mini International Neuropsychiatric Interview (MINI) are being introduced at the NRC.

The rate of prescription medication is also on the low side, consistent with international trends. Moreover, there is a concern over diversion of buprenorphine and more red tape as far as obtaining a license for the NRC to import and use such controlled medications. Other medications like methadone and depot naltrexone are not available in the UAE for treatment of opiate or alcohol addiction.

The data are limited by the fact that this is a retrospective study, based primarily on review of clinical case notes and restricted to only patients seen at the NRC. The data is also limited to adult males. However, recently the NRC opened its first female unit and also plans to open an adolescent unit. In addition, there is no current data on expatriates in the Emirates.

According to available data, there is an increase in the number of admissions for treatment of drug abuse along with an increase in polysubstance and prescription drug abuse particularly since 2009. Considering all the limitations listed above, this is the available set of data from one of the largest and the only comprehensive treatment center in the UAE.

\section{References}

1. World Drug Report [Internet]. New York :United Nations Office on Drugs and Crime;2012 [cited Feb 2012] Available from : http://www.unodc.org/ unodc/en/data-and-analysis/WDR-2012.html 
2. Degenhardt, L. et al. Towards a Global View of Alcohol, Tobacco, Cannabis, and Cocaine Use: Findings from the WHO World Mental Health Surveys. PLoS Med 2008;5(7):1053-1067

3. AbuMadini, M. et al. Two decades of treatment seeking for substance use disorders in Saudi Arabia: Trends and patterns in a rehabilitation facility in Dammam. Drug Alcohol Depend 2008;97:231-236

4. Al-Kandari F.H. et al. Initiation factors for substance abuse. J Adv Nurs 2001;34(1):78-85.

5. Population Estimates 2006-2010 [Internet]. United Arab Emirates: National Bureau of Statistics, 2011 [cited June 2012] Available from: http://www. uaestatistics.gov.ae/ReportDetailsEnglish/tabid/121/Default.aspx?ItemId=1 914\&PTID=104\&MenuId=1

6. Health Statistics 2011 [Internet]. United Arab Emirates: Health Authority Abu Dhabi;2012 [cited June 2012]. Available from: http://www.haad.ae/ HAAD/LinkClick.aspx?fileticket=c-1GoRRszqc\%3d\&tabid=349

7. Amin, Y. et al. Substance abuse consultation rates: Experience from Private Practice in Dubai. Arab J Psychiatr 1996;7(2):133-139

8. Younis, Y.O. et al. A profile of alcohol and drug misusers in an Arab community. Addiction 1995;90:1683-1684

9. Amir, T. et al. Comparison of Patterns of Substance abuse in Saudi Arabia and The United Arab Emirates. Soc Behav Pers 2001;29(6):519-530

10. AlMarri, T.S.K. et al. Alcohol and Substance use in the Arabian Gulf region: A review. Int J Psychol 2009;44(3):222-233

11. Anderson, P. et al. Global use of alcohol, drugs and tobacco. Drug Alcohol Rev 2006;25:489-502

12. Hafeiz, H.B. Socio-demographic correlates and pattern of drug abuse in Eastern Saudi Arabia. Drug Alcohol Depend 1995;38:255-259

13. Al-Nahedh, N. Relapse among substance abuse patients in Riyadh, Saudi Arabia. East Mediterr Health J 1999;5:241-246

14. Rahim, S.A. et al. Long-term Outcome of Treated Addiction in Saudi Arabia: Predictors of Relapse in 10 year Follow-up. Arab J Psychiatr 2005;16(2):93-97 